BERICHTE UBER DIE VERHANDLUNGEN DER SÄCHSISCHEN AKADEMIE DER WISSENSCHAFTEN ZU LEIPZIG

Philologisch-historische Klasse

Bd. $103 \cdot$ Heft 4

KARL BISCHOFF

SPRACHLICHE BEZIEHUNGEN

ZWISCHEN NIEDERDEUTSCHEM ALTLAND

UND NEULAND

IM BEREICH DER MITTLEREN ELBE

Mit 20 Abbildungen

AKA DEMIE V ER LA G B ERLIN

1958 


\section{BERICHTE ÜBER DIE VERHANDLUNGEN DER SÄCHSISCHEN AKADEMIE DER WISSENSCHAFTEN ZU LEIPZIG \\ PAILOLOGISCH·HISTORISCHE KLASSE}

\section{Band 97}

Heft 1 Prof. Dr. Theodor Frnes, Antike und Christentum an der Wiege der deutschen Sprache 36 Selten $-8^{\circ}-1949-$ (vergriffen)

Heft 2 Prof. Dr. FrDadrior Wrutrer, Zum mongolischen Tanjur 36 Seiten $-8^{0}-1949$ - (vergriffen)

Heft 3 Prof. Dr. Waurter Bastrke, Die Götterlehre der Snorra-Edda Nachdruck - 68 Selten $-8^{0}-1952$ - DM 6,30

Heft 4 Prof. Dr. CarL Brookgrimann, Abessinische Studien 60 Seiten $-8^{\circ}-1950-($ vergriffen $)$

Heft 5 Prof. Dr. Whнnelм SoHUBard, Griechische literarische Papyri 108 Seiten $-8^{0}-1950-$ (vergriffen)

Heft 6 Prof. Dr. Franz Dornserfy, Verschmähtes zu Vergil, Horaz und Properz Nachdruck - 108 Seiten $-8^{0}-1951$ - (vergriffen)

Heft 7 Prof. Dr. Werner Kratss, Altspanische Drucke im Besitze der außerspanischen Bibliotheken 112 Seiten $-8^{\circ}-1951$ - (vergriffen)

Heft 8 Prof. Dr. Martin LinTzes, Liebe und Tod bei Heinrich von Kleist 76 Seiten $-8^{0}-1950-$ (vergriffen)

\section{Band 98}

Heft 1 Prof. Dr. FrDEDRIOH ZUOKJr, Freundschaftsbewährung in der neuen attischen Komödie. Ein Kapitel hellenistischer Ethik und Humanität 38 Seiten $-8^{0}-1950-$ (vergriffen)

Heft 2 Prof. Dr. Frnetrion Bren, Vorgeschichtliche Felsbilder in Karelien und West-Sibirien 16 Seiten - 4 Tafeln $-8^{0}-1950$ - (vergriffen)

Heft 3 Dr. JАOOB JATZWAUK, Sorbische Bibliographie, 2. Auflage XX und 500 Seiten $-8^{\circ}-1952-$ (vergriffen)

Heft 4 Prof. Dr. Orro Erssfendr, El im ugaritischen Pantheon 84 Seiten - 1 Tafel als Frontispicium $-8^{0}-1951$ - (vergriffen)

Heft 5 Prof. Dr. Paul Trmesce, Studien zur indogermanischen Wortkunde und Religionsgeschichte Nachdruck -78 Seiten $-8^{0}-1952-$ DM 9,50

Heft 6 Prof. Dr. WAJTER BAETKr, Christliches Lehngut in der Sagareligion. Das Svoldr-Problem Nachdruck - 135 Seiten - $8^{\circ}-1952$ - DM 5,50 
BERICHTE UBER DIE VERHANDLUNGEN DER SÄCHSISCHEN AKADEMIE DER WISSENSCHAFTEN ZU LEIPZIG

Philologisch-historische Klasse

Bd. $103 \cdot$ Heft 4

\author{
KARL BISCHOFF \\ SPRACHLICHE BEZIEHUNGEN \\ ZWISCHEN NIEDERDEUTSCHEM ALTLAND \\ UND NEULAND \\ IM BEREICH DER MITTLEREN ELBE
}

Mit 20 Abbildungen

A K A D E M E - V E R L A G B E R L I N

1958 
Vorgelegt in der Sitzung vom 7. Mai 1956

Manuskript eingeliefert am 16. Mai 1957

Druckfertig erklärt am 25. Januar 1958

Erschienen im Akademie-Verlag GmbH, Berlin W 8, Mohrenstraße 39

Lizenz*Nr. $202 \cdot 100 / 374 / 58 \cdot$ MdI der DDR Nr. 3551

Satz und Druck der Buchdruckerei F. Mitzlaff KG., Rudolstadt/Thür. V/14/7 (2:z)

Bestell- und Verlagsnummer 2026/103/4

Preis: DM 3,60

Printed in Germany 